\title{
EPIDENDRUM TRIPUNCTATUM (ORCHIDACEAE, LAELIINAE): THE TALE OF TWO SPECIES
}

\author{
WeSLEy E. HigGins \\ Marie Selby Botanical Gardens, 811 South Palm Avenue, Sarasota, FL 34236-7726 USA. : \\ whiggins@selby.org.
}

\begin{abstract}
Homonyms can often create confusion and this is the case with Epidendrum tripunctatum. A new combination, Prosthechea micropus, is required to correct the misapplied nomenclature. The author recognizes the Brazilian species as Prosthechea punctifera and the Central American species is recognized as Prosthechea micropus.

RESUMEN. El nombre Epidendrum tripunctatum fue usado por John Lindley para dos especies claramente diferentes; una de México, la otra de Brasil. Los homónimos pueden crear a menudo mucha confusión; en el caso referido es necesaria una nueva combinación, Prosthechea micropus, para corregir la nomenclatura errónea.

Palabras Clave / Key words: Orchidaceae, Laeliinae, homonym, new combination, Epidendrum tripunctatum, Prosthechea tripunctata, Pollardia tripunctata, Encyclia tripunctata, Pseudencyclia tripunctata, Epidendrum punctiferum, Anacheilium punctiferum, Prosthechea punctifera, Epidendrum calamarium, Anacheilum calamarium, Prosthechea micropus.
\end{abstract}

Introduction. When Lindley used the same name for two different species, one Mexican and one Brazilian in Folia Orchidacea (1853), it started a chain of confusion that continues to this day (Table 1). However Lindley is not alone and homonyms abound in taxonomic literature. After examining microfiches of the types at K-Lindl., it is clear that these two uses of Epidendrum tripunctatum Lindl. represent two different entities, although both would be included in Encyclia s.1. (Dressler 1961). The following combinations may be validly published, yet incorrect, as they are based on later and wrong homonyms:

Prosthechea tripunctata (Lind1.) W. E. Higgins, Phytologia 82: 381, 1997.

Pollardia tripunctata (Lindl.) Withner \& Harding, Cattleyas \& Relatives: Debatable Epidendrums, 244, 2004.

\section{The Mexican Plant}

John Lindley (1841) described a very distinctive species that he received from Richard Harrison, Esq. of Aighburgh, which was most likely of Mexican or Central American origin. Lindley described the plant as having long grassy leaves and a very short twoflowered scape. The sepals are twice as broad and much larger than the petals; both of them are widely spreading and a dull yellow green. The column is deep purple, with three lobes, each of which is tipped with one pale orange-colored spot. The lip is rather more than half an inch long, obovate, rugose, without being warted, and distinctly emarginate near the apex of the column; its color is pale citron. This plant (Fig. 1) was clearly the species we know today from Mexico. Dressler (1961) transferred the Mexican species to Encyclia tripunctata (Lindl.) Dressler. Higgins (1997) attempted to transfer the Mexican plant to Prosthechea but incorrectly cited the Brazilian homonym. Withner and Harding (2004) illustrated the Mexican species but cited the Brazilian basionym when they proposed the genus Pollardia. This leaves the Mexican species without a valid Prosthechea combination (or in Pollardia for that matter).

\section{New Nomenclature}

Prosthechea micropus (Rchb.f.) W.E. Higgins comb. nova

Basionym: Epidendrum micropus Rchb. f. Hamburger Garten- und Blumenzeitung 20: 13. 1863. Type: Borsig s.n., cult. (W).

Synonyms: Encyclia tripunctata (Lindl.) Dressler, 
Table 1. Comparison of nomenclature for the two species.

\begin{tabular}{|c|c|c|}
\hline & Mexican Plant & Brazilian Plant \\
\hline Current Name & $\begin{array}{l}\text { Prosthechea micropus (Rchb.f.) W.E.Higgins, } \\
\text { Lankesteriana 4(3): 223. } 2004 .\end{array}$ & $\begin{array}{l}\text { Prosthechea punctifera (Rchb.f.) W.E.Higgins, } \\
\text { Phytologia 82: } 381.1997 \text { (1998). }\end{array}$ \\
\hline Basionym & $\begin{array}{l}\text { Epidendrum micropus Rchb.f. Hamburger Garten- } \\
\text { Blumenzeitung 20: 13. } 1863 .\end{array}$ & $\begin{array}{l}\text { Epidendrum punctiferum Rchb.f., Gard. Chron. } \\
\text { n.s. 16: 38. } 1881 .\end{array}$ \\
\hline Original Description & $\begin{array}{l}\text { Epidendrum tripunctatum Lindl., Edwards's Bot. } \\
\text { Reg. 27: } 66.1841 .\end{array}$ & $\begin{array}{l}\text { Epidendrum tripunctatum Lindl., Fol. Orchid. } \\
\text { Epidendrum 41. } 1853 .\end{array}$ \\
\hline Other Synonyms & $\begin{array}{l}\text { Epidendrum diguetii Ames, Sched. Orch. 1: } 15 . \\
1922 . \\
\text { Encyclia diguetii (Ames) Hoehne, Arq. Bot. } \\
\text { Estado São Paulo, nova ser., f. maior, 2: } 151 . \\
1952 . \\
\text { Encyclia tripunctata (Lindl.) Dressler, Brittonia 13: } \\
\text { 265. } 1961 . \\
\text { Pseudencyclia tripunctata (Lindl.) V.P. Castro \& } \\
\text { Chiron, Richardiana 4(1): } 33.2003 .\end{array}$ & $\begin{array}{l}\text { Encyclia punctifera (Rchb.f.) Pabst, Orquídea } \\
\text { (Rio de Janeiro) 29(6): 277. } 1967 \text { (1972). } \\
\text { Anacheilium punctiferum (Rchb.f.) F.Barros, } \\
\text { Hoehnea 10: 85. } 1983 \text { (1984). } \\
\text { Prosthechea punctifera (Rchb.f.) W.E.Higgins, } \\
\text { Phytologia 82: 380. } 1997 \text { (1998). } \\
\text { Prosthechea tripunctata (Lindl.) W.E.Higgins, } \\
\text { Phytologia 82: } 381.1997 \text { [nom. err.] } \\
\text { Pollardia tripunctata (Lindl.) Withner \& Harding, } \\
\text { Cattleyas \& Relatives: Debatable Epiden- } \\
\text { drums 244. } 2004 \text { [nom. confus.] }\end{array}$ \\
\hline
\end{tabular}

Brittonia 13: 265. 1961.

Encyclia diguetii (Ames) Hoehne, Arq. Bot. Estado São Paulo 2: 151. 1952.

Epidendrum diguetii Ames Sched. Orchid. No. 1: 15. 1922.

Epidendrum tripunctatum Lindl., Edwards's Bot Reg. 27: 66, 1841 [non Lindl. 1853].

Pseudencyclia tripunctata (Lindl.) V.P. Castro \& Chiron, Richardiana 4(1): 33, 2003.

Note: The epithet "micropus" is a noun in apposition that remains "micropus" even under a feminine generic name. Another example of an indeclinable noun is "saxicola" therefore Orthophytum saxicola (Bromeliaceae).

\section{The Brazilian Plant}

Lindley (1853) described a Brazilian species using the same name Epidendrum tripunctatum. This plant collected in the wilds of Brazil and sent to Loddiges, was described as being much like Epidendrum calamarium Lindl. in form (fig. 2). Flowers yellowish, with three deep purple spots in the front of the calli, and two or three smaller on each side above the furrows of the lip. Reichenbach $f$. recognized the homonymy and published a new name for the second "E. tripunctatum" as Epidendrum punctiferum Rchb.f. in 1881. Pabst (1967) transferred the Brazilian species to Encyclia followed by Barros (1983) who transferred it to Anacheilium. When
Higgins (1997) resurrected Prosthechea, he included the Brazilian species. The Withner and Harding combination and the Higgins combination based on Epidendrum tripunctatum Lindl., Fol. Orchid. Epidendrum 41, 1853 are superfluous. Withner and Harding include Epidendrum punctiferum Rchb.f. as a synonym of Anacheilium calamarium (Lindl.) Pabst, Moutinho \& A.V.Pinto when E. punctiferum is a replacement name for E. tripunctatum (1853). Higgins treats Epidendrum punctiferum Rchb.f. as Prosthechea punctifera (Rchb.f.) W.E.Higgins.

\section{TAXONOMIC TREATMENT}

Prosthechea punctifera (Rchb.f.) W.E.Higgins, Phytologia 82: 380, 1997 (1998).

Basionym: Epidendrum punctiferum Rchb.f., Gard. Chron. n.s. 16: 38, 1881.

Synonyms: Anacheilium punctiferum (Rchb.f.) F.de Barros, Hoehnea 10: 85, 1983 (1984).

Encyclia punctifera (Rchb.f.) Pabst, Orquidea (Rio de Janeiro) 29(6): 277. 1967 (1972).

Epidendrum tripunctatum Lindl. [nom. illeg.] Fol. Orchid. Epidendrum 41, 1853, non Lindl. 1841.

Pollardia tripunctata (Lind1.) Withner \& Harding, Cattleyas and their Relatives, 244, 2004 [nom. confus.]

Prosthechea tripunctata (Lindl.) W. E. Higgins, Phytologia 82: 381, 1997 (1998). [nom. err.] 

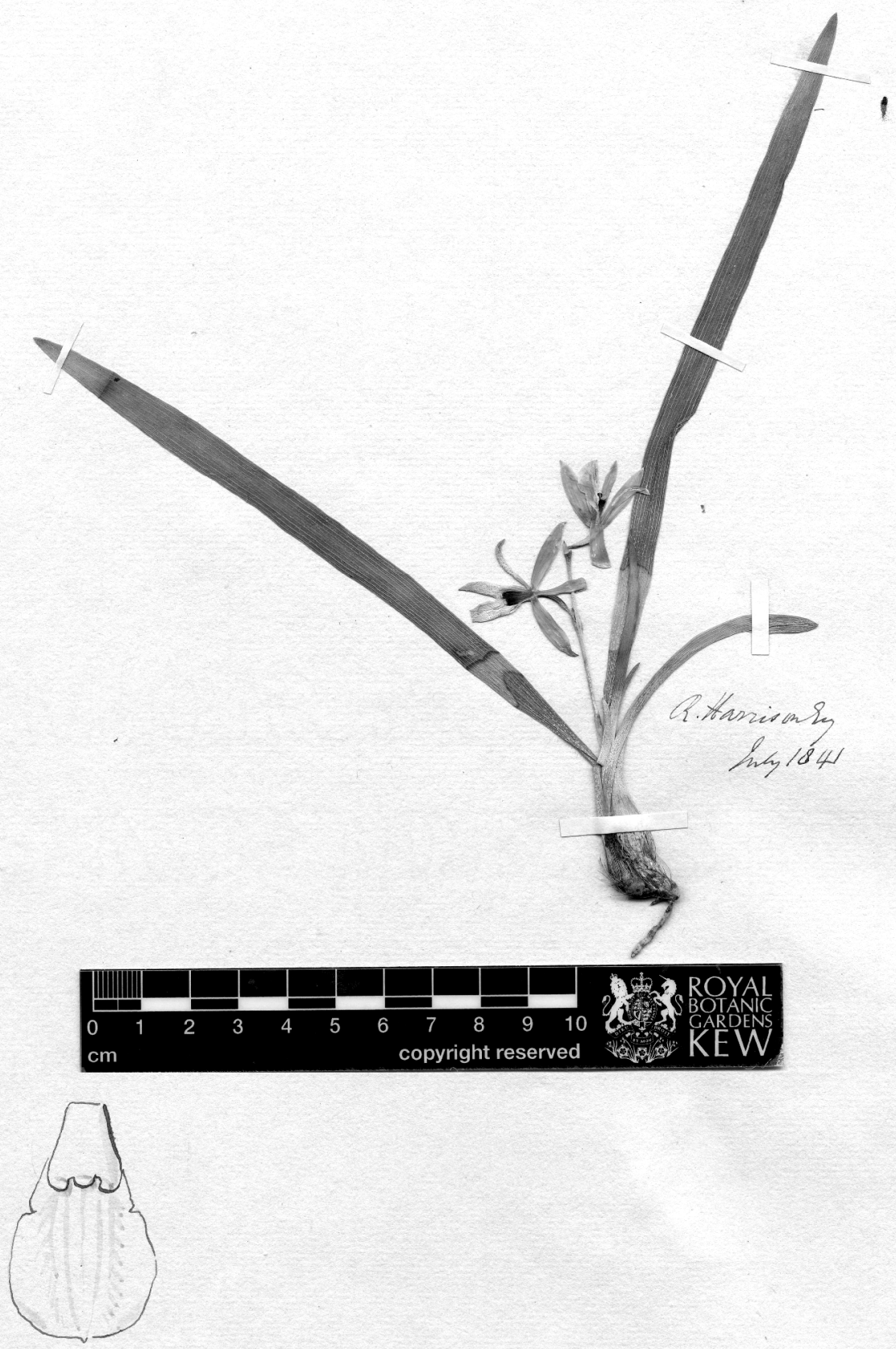

ROYAL BOTANIC GARDENS KEW

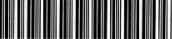

K000079062

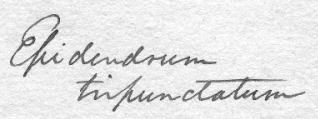

Fig. 1. Epidendrum tripunctatum Lindl. 1841 [Type: K-Lindl.] Reproduced with the kind permission of the Director and Trustees of the Royal Botanic Gardens, Kew. 


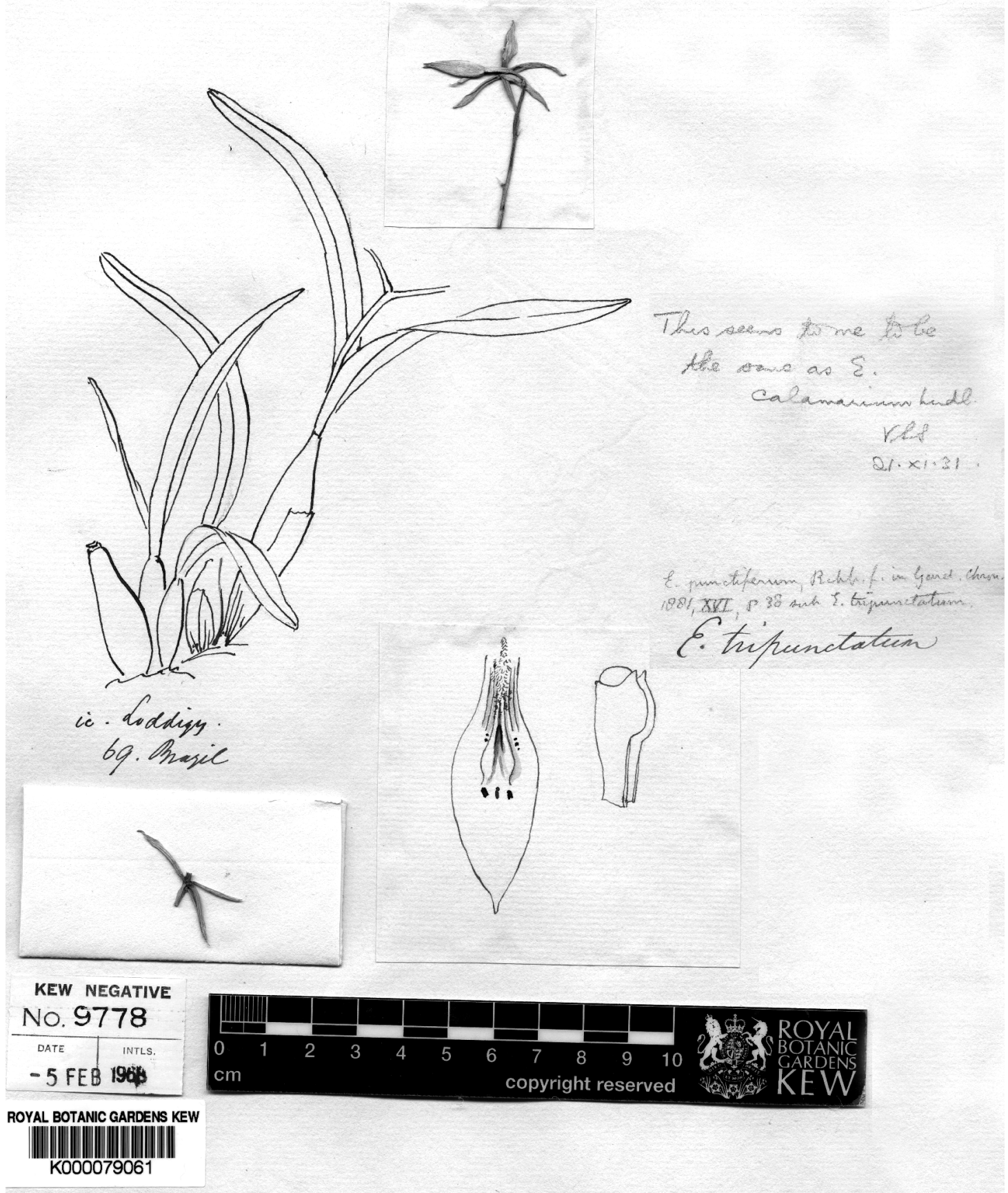

Fig. 2. Epidendrum tripunctatum Lindl. 1853 [Type: K-Lindl.] Reproduced with the kind permission of the Director and Trustees of the Royal Botanic Gardens, Kew. 

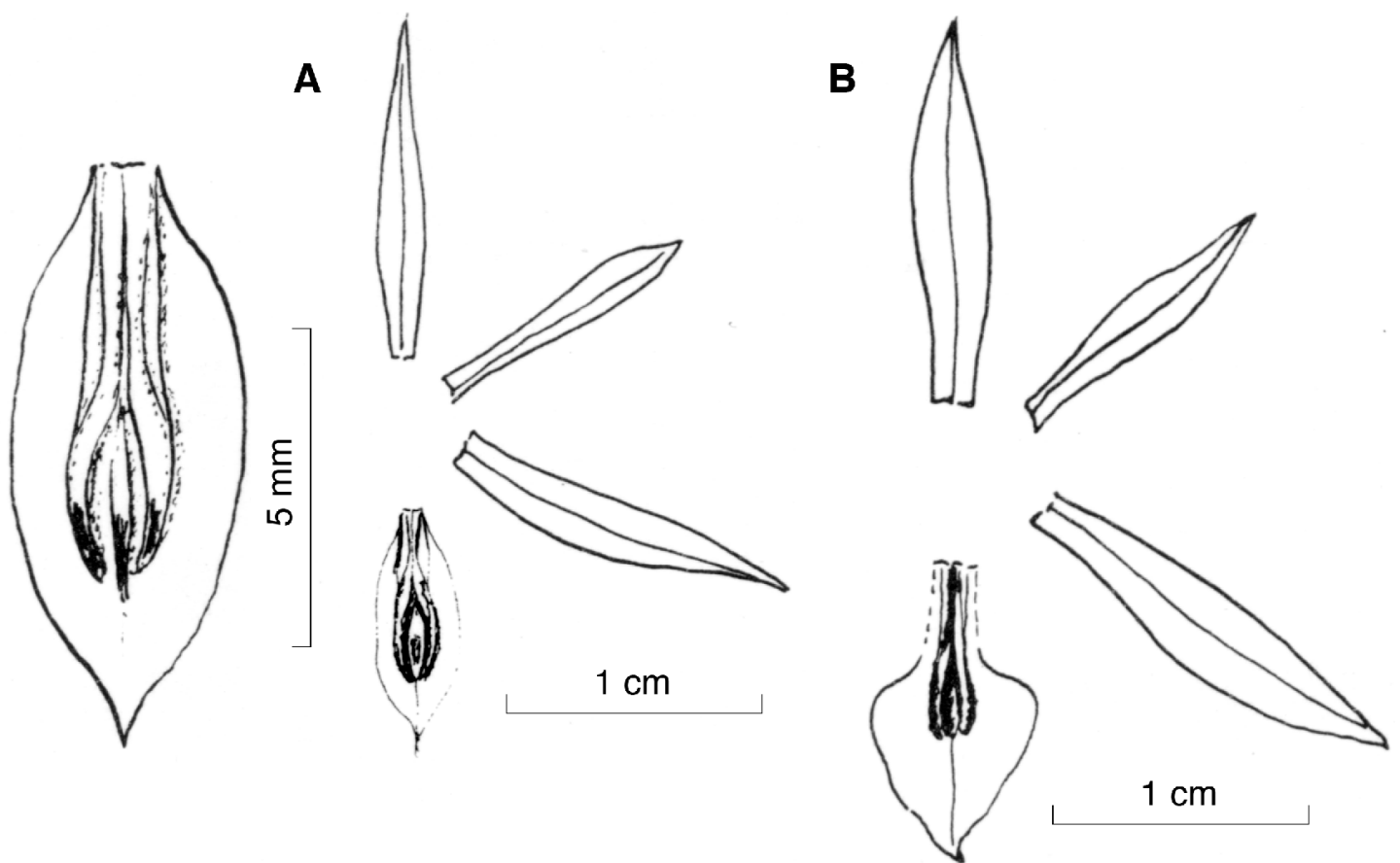

Fig. 3. Drawings of: A. Prosthechea punctifera (No. 683) and B. Prosthechea calamaria (No. 679) from Pabst \& Dungs, I:300, 1977.

\section{Chronological}

\section{NOMENCLATURALly IMPORTANT CitATIONS}

Epidendrum tripunctatum Lindl. Edwards' Bot. Reg. 27: 66, 1841.

Epidendrum tripunctatum Lindl. Fol. Orchid. Epidendrum 41, 1853. [nom. illeg.]

Epidendrum punctiferum Rchb.f., Gard. Chron. n.s. 16: 38, 1881.

Basionym: Epidendrum tripunctatum Lindl., Fol. Orchid. Epidendrum 41, 1853, non Lindl. 1841.

Encyclia tripunctata (Lind1.) Dressler, Brittonia 13: 265, 1961.

Basionym: Epidendrum tripunctatum Lindl., Edwards's Bot Reg. 27: 66, 1841 [non Lindl. 1853].

Encyclia punctifera (Rchb.f.) Pabst. Orquidea (Rio de Janeiro) 29(6): 277. 1967 (1972).

Basionym: Epidendrum punctiferum Rchb.f., Gard. Chron. n.s. 16: 38, 1881.

Anacheilium punctiferum (Rchb.f.) F. Barros, Hoehnea 10: 85, 1983 (1984)

Basionym: Epidendrum punctiferum Rchb.f., Gard. Chron. n.s. 16: 38, 1881 .

Prosthechea punctifera (Rchb.f.) W.E.Higgins, Phytologia 82: 380, 1997 (1998).

Basionym: Epidendrum punctiferum Rchb.f., Gard. Chron. 2: 38. 1881.
Prosthechea tripunctata (Lind1.) W. E. Higgins, Phytologia 82: 381, 1997 (1998).

Basionym: Epidendrum tripunctatum Lindl., "Fol. Orchid. Epidendrum 41, 1841 [sic 1853]".

Pseudencyclia tripunctata ( Lindl. ) V.P.Castro \& Chiron. Richardiana 4(1): 33.2003

Basionym: Epidendrum tripunctatum Lindl. Edward's Bot. Reg. 27: misc. 66. 1841.

Pollardia tripunctata (Lind1.) Withner \& Harding, Cattleyas \& Relatives: Debatable Epidendrums, 244, 2004.

Basionym: Epidendrum tripunctatum Lindl., Fol. Orchid. Epidendrum 41, 1853.

\section{Related taxonomic treatment}

Withner and Harding (2004) cite Epidendrum punctiferum Rchb.f. under the synonymy of Anacheilum calamarium (Lindl.) Pabst. When I examine the Lindley drawing on the type specimen sheet I find a number of differences in lip and callus shape. Thus it is my opinion that Prosthechea calamaria and $P$. punctifera are two distinct species (Fig. 3).

Prosthechea calamaria (Lind1.) W.E.Higgins, Phytologia 82: 377. 1997 (1998). 
Basionym: Epidendrum calamarium Lindl. Bot. Reg. 24 misc. 88, n. 163. 1838.

Synonyms: Anacheilium calamarium (Lindl.)

Pabst, Moutinho \& A.V.Pinto, Bradea 3(23):

183. 1981

Encyclia calamaria (Lindl.) Pabst, Orquidea (Rio de Janeiro) 29(6): 276. 1967 (1972)

Hormidium calamarium (Lindl.) Brieger,

Publicação Cientifica Universidade de São

Paulo, Instituto de Genetica 2:69. 1961.

ACKNOWLEDGEMENTS. I thank Kanchi Gandhi and Gustavo A. Romero for bringing these errors to my attention, David Roberts and Board of Trustees of RBG Kew for the photos of the Lindley specimens, Walter Till for Latin assistance, and Robert Dressler and two anonymous reviewers for comments on the manuscript.

\section{LITERATURE CITED}

Barros, F. 1983 (1984). Flora fanerogâmica da reserva do parque estadual das Fontes do Ipiranga (São Paulo, Barsil). Hoehnea 10: 74-124.

Dressler, R. L. 1961. A reconsideration of Encyclia (Orchidaceae). Brittonia, 13: 253-266.

Higgins, W. E. 1997 (1998). A reconsideration of the genus Prosthechea (Orchidaceae). Phytologia 82: 370-383.

Lindley, J. 1841. Epidendrum tripunctatum. Edwards's Bot Reg. 27: 66.

Lindley, J. 1853. Folia Orchidaceae: Epidendrum. London: Bradbury and Evans, Printers, Whitefriars.

Pabst, G.F.J. 1967 (1972). Encyclia punctifera. Orquidea (Rio de Janeiro) 29(6): 277.

Pabst, G.F.J. \& F. Dungs. 1977. Orchidaceae Brasilienses. Gesamtherstellung: Hagemann-Druck, Hildesheim.

Withner, C.L. \& P.A. Harding. 2004. The Cattleyas and Their Relatives: The Debatable Epidendrums. Timber Press, Portland. 